\title{
Effectiveness of highly active antiretroviral therapy among HIV-1 infected women
}

\section{S J Gange, Y Barrón, R M Greenblatt, K Anastos, H Minkoff, M Young, A Kovacs, M Cohen, W A Meyer III, A Muñoz for the Women's Interagency HIV Study Collaborative Study Group}

J Epidemiol Community Health 2002;56:153-159

See end of article for authors' affiliations

\section{Correspondence to:} Dr S J Gange, Department of Epidemiology, Johns Hopkins School of Hygiene and Public Health, $615 \mathrm{~N}$ Wolfe Street, Room E-7010, Baltimore, MD 21205, USA

sgange@jhsph.edu

Accepted for publication 3 July 2001

\begin{abstract}
Study objective: To describe the impact of highly active antiretroviral therapy (HAART) on mortality, morbidity, and markers of HIV disease progression in HIV infected women.

Design: Data collected from the Women's Interagency HIV Study, a prospective cohort study that enrolled women between October 1994 and November 1995.

Setting: Six clinical consortia based in five cities in the United States (New York, NY; Washington, DC; Los Angeles, CA; San Francisco, CA; and Chicago, IL).

Participants: A total of 1691 HIV seropositive women with a study visit after April 1996.

Main results: Beginning in April 1996, the self reported use of HAART increased over time, with more than $50 \%$ of the cohort reporting HAART use in 1999 . There was a $23 \%$ decline per semester in the incidence of AIDS from April 1996 (95\% confidence intervals (CI) -29\% to - 16\%). Furthermore, there was a $21 \%$ decline of the semiannual mortality rates among those with AIDS at baseline $195 \% \mathrm{CI}-27 \%$ to $-14 \%)$ and an $11 \%$ decline among those AIDS free at baseline $(95 \% \mathrm{Cl}-3 \%$ to $-18 \%)$. CD4+ lymphocyte counts either increased (women with baseline AIDS) or stabilised (women without baseline AIDS) after April 1996, and HIV RNA levels dramatically declined in both groups, although the percentage of women with HIV RNA above $4000 \mathrm{cps} / \mathrm{ml}$ remained stable at approximately $40 \%$ since mid-1997.
\end{abstract}

Conclusions: Despite concerns regarding the use of antiretroviral therapies in this population, the use of therapies led to improved immunological function, suppressed HIV disease activity, and dramatic declines in morbidity and mortality.
$\mathrm{B}$ eginning in the mid-1990s, significant advances have been made in the treatment of human immunodeficiency virus type 1 (HIV) infection, and new antiretroviral nucleoside reverse transcriptase inhibitors (RTIs) are now routinely used in combination with potent protease inhibitors and non-nucleoside RTIs. The efficacy of these highly active antiretroviral therapies (HAART) has been demonstrated in numerous clinical trials, ${ }^{1-3}$ but observational studies of HIV infected populations provide the opportunity to supplement and complement these findings in two ways. ${ }^{4}$ Firstly, similar to clinical trial analyses, observational data can be used to compare outcomes among individuals reporting and not reporting use of therapies. These analyses provide measures of individual effectiveness among a population that is generally larger, followed up for a longer duration, and comprised of participants who are more representative of existing patient populations. Therapies in observational studies, however, are not randomised as in clinical trials, and their use is dependent on a variety of demographic ${ }^{56}$ and health related factors that are indications for treatment. ${ }^{7}$ These analyses, while important for supplementing the findings of clinical trials, are therefore vulnerable to residual confounding because of unmeasured factors leading to the use or prescription of various therapies, and sophisticated multivariate analyses are required to draw correct inferences. ${ }^{8-12}$

In contrast with measuring the effect of therapies at the individual level, a second approach is to estimate the effectiveness of therapies at the population level. This approach measures the impact of an intervention by examining the reduction of mortality or changes in markers before and after its introduction. Population effectiveness in cohort studies capitalises on the longitudinal collection of data and compares cohort outcomes in different time periods. ${ }^{43-15}$ This analysis requires that trends in the use of treatment and other relevant factors be carefully documented to rule out alternative explanations for observed differences in the outcomes under study, and is most convincing when new therapies become available and widely used at an identifiable point in time. The comparisons in the occurrence of disease and trends in disease markers provide a relevant and important public health perspective: effectiveness measures the overall changes in population health, reflecting the net effect of all factors that influence access, prescription, efficacy, adherence, discontinuation and development of potential resistance to therapies.

A number of prior studies have documented the effectiveness of different HIV therapies in reducing mortality and the incidence of AIDS in different populations ${ }^{11}{ }^{16-22}$ as well as increasing the time after an AIDS diagnosis. ${ }^{23}{ }^{24} \mathrm{Few}$ of these studies, however, have focused specifically on the long term effectiveness of HAART in women and ethnic minorities, the populations in which HIV has expanded in recent years in the United States. ${ }^{25-28}$ Epidemiological analyses have shown that women and minorities now represent $67 \%$ of newly diagnosed AIDS cases, $62 \%$ of people living with AIDS, and $69 \%$ of newly reported diagnoses of HIV infection. ${ }^{27}{ }^{28}$ Differences in the response to HAART among these populations has been reported by some groups ${ }^{29}{ }^{30}$ but not others. ${ }^{23}{ }^{24}$ The basis for these differences is likely to be multifactorial. Firstly, the response to HAART may be different because of biological factors. Viral load differences have been documented between

Abbreviations: HAART, highly active antiretroviral therapy; RTIs, reverse transcriptase inhibitors; ART, antiretroviral therapy; WIHS, Women's Interagency HIV Study 
men and women at higher CD4+ lymphocyte counts, ${ }^{31-33}$ although the physiological basis for these differences is unclear. Gender-based differences in either viral replication rates or decay rates may explain these viral load differences, as the steady state of viral load is reached rapidly. ${ }^{34}$ If these differences exist, they may have implications for how effective therapies are at reducing viral levels in women compared with men. One possible explanation for the gender-based differences is the difference in hormonal milieu. Sex steroids such as oestrogens and progestins are important modulators of immunological activity and thus could plausibly influence host responses to HIV infection and HIV replication rate. Greenblatt et al ${ }^{36}$ have recently reported the influence of ovulatory cycle phase on plasma HIV RNA level, with significantly lower levels occurring in the midluteal compared with the early follicular cycle phase.

Furthermore, HIV infected women and minorities are more likely to have a lower socioeconomic status, ${ }^{37}$ which may have a number of consequences affecting the effectiveness of HAART, including lower adherence to treatment ${ }^{38}$ and different utilisation patterns of antiretroviral therapy (ART). ${ }^{39}$ Access to health care may also play an important part, ${ }^{39}{ }^{40}$ because health coverage is not universal in the United States and dependent on access to public and private health insurance. However, it has been shown that the Ryan White CARE Act of 1990, a federal assistance programme whose goal is to help HIV infected people overcome financial barriers to accessing HIV medical care, has seemed to equalise access to medical services for women and minorities. ${ }^{41}$

In this report, we investigate the effectiveness of HAART in the Women's Interagency HIV Study (WIHS), an ongoing cohort study of HIV infected women and women at risk for HIV infection. The demographics of the WIHS cohort closely mirror the characteristics of HIV infected women in the general US population ${ }^{42}$ and clinical care is provided in a variety of settings. A more complete discussion of the trends in ART and predictors of HAART usage in the WIHS have been presented elsewhere. ${ }^{57}$ In this report, we document longitudinal trends in mortality, self reported clinical AIDS, and markers of HIV disease progression (CD4+ lymphocyte count, and plasma HIV RNA) in this representative cohort, to contribute to the understanding of the public health impact of these new potent treatments.

\section{METHODS}

\section{Subjects and Design}

The WIHS is an ongoing prospective study of HIV-1 infection in women, conducted in five locations within the United States: New York City (two sites), Washington DC, Chicago, Southern California and the San Francisco Bay area. The WIHS methods and baseline cohort characteristics have been described previously. ${ }^{42}$ Briefly, from October 1994 to November 1995, 2628 women (2059 HIV- I seropositive and 569 seronegative) were enrolled in WIHS. Women were recruited from HIV primary care clinics, hospital-based programmes, research programmes, community outreach sites, women's support groups, drug rehabilitation programmes, HIV testing sites, and referrals from enrolled participants. The HIV infected and uninfected women were recruited from similar sources and were matched on demographic and risk factors for acquisition of HIV infection. Every six months, WIHS participants were interviewed using a structured questionnaire, received physical and gynaecological examinations, and provided multiple laboratory specimens. In this report, AIDS defining clinical conditions (consistent with 1993 CDC clinical surveillance conditions, but excluding immunological based criteria of low CD4), ${ }^{43}$ were ascertained through self report. Participants' deaths were ascertained continuously using a combination of active (for example, death certificate abstraction upon notification of a participant's death) and passive (for example,

\section{Key points}

- Population effectiveness is an important public health measure of the impact of treatments and complements findings from clinical trials.

- Newly available treatments have significantly affected the manifestations of HIV-1 infection in the Women's Interagency HIV Study, a population-based prospective cohort study.

- Continued monitoring of the Women's Interagency HIV Study will be important for future trends in HIV disease outcomes as new treatments are approved.

national death registry searches) surveillance methods. After five years, the overall retention rate in the WIHS was approximately $81 \%{ }^{44}$

Quantification of HIV-l RNA in plasma was performed using the isothermal nucleic acid sequence based amplification (NASBA/Nuclisens) method (Organon Teknika, Durham, NC) in laboratories participating in the NIH/NIAID, Virology Quality Assurance Laboratory proficiency testing programme. The lower limit of quantification through 9/97 was 4000 copies/ml using a $0.1 \mathrm{ml}$ sample input; from 10/97 through 12/98 the lower limit was 400 copies $/ \mathrm{ml}$ using $0.2 \mathrm{ml}$ sample input; after 1/99 the lower limit was 80 copies $/ \mathrm{ml}$ using $1.0 \mathrm{ml}$ sample input. Lymphocyte subsets were quantified using standard flow cytometric methods in laboratories participating in the NIH/NIAID Flow Cytometry Quality Assessment Program.

At each study visit, self reported ART use in the period since the previous visit was assessed by interviewers stating the name of each drug, both by brand and generic drug name, and showing participants photomedication cards. For this analysis, we focused on the three classes of FDA approved therapies: nucleoside RTIs, including zidovudine, stavudine, zalcitabine, didanosine, and lamivudine; protease inhibitors, including saquinavir, indinavir, ritonavir, and nelfinavir; and nonnucleoside RTIs, including nevirapine and delavirdine. HAART was defined according to the 1997 Department of Health and Human Services guidelines ${ }^{45}$ as two or more nucleoside RTIs with either a protease inhibitor or a non-nucleoside RTI. Women taking a protease inhibitor plus zidovudine and stavudine, however, were classified as receiving non-HAART combination therapy, as indicated by the treatment guidelines.

Because of the interest in evaluating longitudinal trends in immunological and virological markers surrounding the introduction of HAART, we restricted our analysis of markers to HIV seropositive participants who had at least one WIHS study visit after 1 April 1996. This date was chosen as the approximate time when HAART became available to women in this cohort. With this restriction, we minimise capturing any longitudinal trends in markers that occurred because of cohort drop out or death before HAART was available.

\section{Statistical analysis}

Data were categorised into six month calendar time intervals: 10/94-3/95, 4/95-9/95, 10/95-3/96, 4/96-9/96, 10/96-3/97, $4 / 97-9 / 97,10 / 97-3 / 98,4 / 98-9 / 98$, and 10/98-3/99. The incidence of mortality and AIDS defining illnesses (self reported) was computed for each time interval and was constructed from the number of deaths or reported AIDS cases divided by the total number of person years of observation in the six month period. The temporal trends in the incidence of AIDS and death were evaluated using log-linear (Poisson) regression models. ${ }^{46}$

Longitudinal changes in CD4+ and CD8+ lymphocyte counts through 9/99 were modelled using linear random effects regression. ${ }^{47}$ Segmented linear regression models were used to facilitate comparisons of marker trajectories before 
Table 1 Baseline characteristics of WIHS participants with at least one study visit after 1 April 1996. Characteristics that do not sum to category totals are because of missing values

\begin{tabular}{|c|c|c|}
\hline & $\begin{array}{l}\text { Self reported AIDS at } \\
\text { baseline }(n=492)\end{array}$ & $\begin{array}{l}\text { No self reported AIDS at } \\
\text { baseline }(n=1199)\end{array}$ \\
\hline Median age in years (range) & $38(20-62)$ & $36(17-73)$ \\
\hline \multicolumn{3}{|l|}{ Race/ethnicity } \\
\hline White & 95 (19\%) & $205(17 \%)$ \\
\hline Black & $277(56 \%)$ & $666(56 \%)$ \\
\hline Hispanic & $108(22 \%)$ & $295(25 \%)$ \\
\hline Other & $12(3 \%)$ & $30(2 \%)$ \\
\hline Education less than high school & $185(38 \%)$ & $446(37 \%)$ \\
\hline \multicolumn{3}{|l|}{ Annual household income } \\
\hline$\leqslant \$ 12000$ & $318(66 \%)$ & $717(62 \%)$ \\
\hline \multicolumn{3}{|l|}{ Risk category } \\
\hline Injection drug use & $199(41 \%)$ & $378(32 \%)$ \\
\hline Heterosexual risk & $181(37 \%)$ & $510(43 \%)$ \\
\hline Transfusion risk & $23(5 \%)$ & $42(3 \%)$ \\
\hline Risk not unidentified & $82(17 \%)$ & $258(22 \%)$ \\
\hline \multicolumn{3}{|c|}{ CD4+ lymphocyte counts (cells $/ \mathrm{mm}^{3}$ ) } \\
\hline Mean (standard deviation) & $336.1(272.1)$ & $429.9(281.4)$ \\
\hline$<200$ & $171(36 \%)$ & $224(19 \%)$ \\
\hline $200-500$ & $195(41 \%)$ & $573(49 \%)$ \\
\hline$\geqslant 500$ & $107(23 \%)$ & $364(31 \%)$ \\
\hline \multicolumn{3}{|l|}{ HIV-1 RNA (copies/ml) } \\
\hline $\begin{array}{l}\text { Median (interquartile range) } \\
\leqslant 4000\end{array}$ & $\begin{array}{l}31500(5500-130000) \\
370(77 \%)\end{array}$ & $\begin{array}{l}14000(<4000-62500) \\
762(65 \%)\end{array}$ \\
\hline
\end{tabular}

and after the time when HAART was introduced in the cohort (4/96). That is, linear models were used to summarise marker trajectories over time, but the slopes of the markers obtained before 4/96 were allowed to be different from the slopes of the markers obtained after 4/96. We also investigated models for square root transformed CD4+ and CD8+ lymphocyte counts, but the results were similar to those obtained using the untransformed data. Because of the relatively high limit of plasma HIV RNA quantification $(4000 \mathrm{cps} / \mathrm{ml})$ in early samples, we analysed trends in the prevalence of detectable HIV RNA (>4000 cps/ml). Generalised estimating equation (GEE) methods ${ }^{48}$ were used with a binary response of whether HIV RNA was detectable and with time as a continuous predictor. Similar to the lymphocyte data, the observed slopes in the prevalence of undetectable HIV RNA before and after 4/96 were compared while adjusting for the correlation among repeated measurements from the same person.

\section{RESULTS}

Of the 2059 HIV positive women enrolled in the WIHS, 692 reported AIDS defining clinical conditions at their baseline visit (10/94-11/95). Seventy one per cent of the women with clinical AIDS at baseline $(n=492)$, and $88 \%$ of the women without clinical AIDS at baseline $(n=1199)$, participated in a study visit after 1 April 1996. Table 1 describes selected baseline demographic characteristics of these women. The median age of the cohort was in the mid-30s, and a majority of participants were African-American and with an annual household income less than \$12 000. Most women reported risk exposures from either injecting drugs or through heterosexual contact (with a man at high risk for HIV infection). Women reporting AIDS conditions at baseline had lower CD4+ lymphocyte counts and higher HIV RNA levels.

At enrollment, approximately $35 \%$ of HIV positive women reported current use of any prescription ART, which for the majority comprised monotherapy (78\%). During the final period under study (3/99-9/99), reported current use of any ART increased to $69 \%$ overall. With the introduction of protease inhibitors and non-nucleoside RTIs, the number of women reporting current use of HAART between 3/99 and 9/99 increased to approximately $50 \%$ (table 2). The majority of women on HAART reported use of one protease inhibitor with

Table 2 Longitudinal trends of reported use of highly active antiretroviral therapy (HAART), incidence of self reported clinical AIDS, and mortality

\begin{tabular}{|c|c|c|c|c|c|}
\hline \multirow[b]{2}{*}{ Time period } & \multicolumn{2}{|c|}{ Number reporting use of HAART (\%) } & \multirow[b]{2}{*}{ AIDS incidence rate* } & \multicolumn{2}{|l|}{ Mortality rate* } \\
\hline & AIDS at baseline & $\begin{array}{l}\text { AIDS free at } \\
\text { baseline }\end{array}$ & & AIDS at baseline & $\begin{array}{l}\text { AIDS free at } \\
\text { baseline }\end{array}$ \\
\hline $4 / 95-9 / 95$ & $5(1.1 \%)$ & $4(0.4 \%)$ & $12.09(57 / 471.4)$ & $19.47(41 / 210.6)$ & $2.19(11 / 502.8)$ \\
\hline $10 / 95-3 / 96$ & $8(1.8 \%)$ & $9(0.8 \%)$ & $13.74(74 / 538.7)$ & $23.22(59 / 254.1)$ & $4.96(30 / 604.3)$ \\
\hline $4 / 96-9 / 96$ & $44(9.3 \%)$ & $85(7.1 \%)$ & $14.50(72 / 496.4)$ & $16.92(40 / 236.4)$ & $3.58(21 / 586.8)$ \\
\hline $10 / 96-3 / 97$ & $133(30.2 \%)$ & $261(25.1 \%)$ & $12.85(58 / 451.4)$ & $12.18(27 / 221.7)$ & $4.24(24 / 565.9)$ \\
\hline $4 / 97-9 / 97$ & $163(39.6 \%)$ & $387(37.3 \%)$ & $8.48(36 / 424.3)$ & $11.35(24 / 211.4)$ & $2.91(16 / 548.9)$ \\
\hline $10 / 97-3 / 98$ & $189(49.2 \%)$ & $428(42.2 \%)$ & $6.23(25 / 401.0)$ & $9.69(19 / 196.0)$ & $3.03(16 / 528.4)$ \\
\hline 4/98-9/98 & 159 (43.9\%) & $415(43.7 \%)$ & $4.47(17 / 380.1)$ & $5.90(11 / 186.4)$ & $2.54(13 / 511.0)$ \\
\hline $10 / 98-3 / 99$ & $167(49.9 \%)$ & $479(51.2 \%)$ & $5.07(18 / 354.9)$ & $6.32(11 / 174.0)$ & $2.46(12 / 486.9)$ \\
\hline Overall change per semester & & & $-14 \%$ & $-17 \%$ & $-5 \%$ \\
\hline $95 \% \mathrm{Cl}$ & & & $-18 \%$ to $-10 \%$ & $-22 \%$ to $-12 \%$ & $-12 \%$ to $+2 \%$ \\
\hline Change per semester after $4 / 96$ & & & $-23 \%$ & $-21 \%$ & $-11 \%$ \\
\hline $95 \% \mathrm{Cl}$ & & & $-29 \%$ to $-16 \%$ & $-27 \%$ to $-14 \%$ & $-18 \%$ to $-3 \%$ \\
\hline
\end{tabular}


Table 3 Mean level and annual change in CD4+ and CD8+ lymphocyte counts before and after $4 / 96.95 \%$ Confidence intervals are given in parentheses

\begin{tabular}{|c|c|c|}
\hline & $\begin{array}{l}\text { Self reported AIDS at } \\
\text { baseline }\end{array}$ & $\begin{array}{l}\text { No self reported AIDS at } \\
\text { baseline }\end{array}$ \\
\hline \multicolumn{3}{|c|}{ CD4+ lymphocyte count (cells $/ \mathrm{mm}^{3}$ ) } \\
\hline Mean level in 4/96 & $310.2(288.2$ to 332.1$)$ & 404.8 (390.3 to 419.3 ) \\
\hline Slope per year $10 / 94-4 / 96$ & $-19.4(-32.9$ to -5.9$)$ & $-13.0(-22.2$ to -3.7$)$ \\
\hline Slope per year 4/96-9/99 & $7.2(2.7$ to 11.7$)$ & $1.1(-2.0$ to 4.1$)$ \\
\hline Difference of slopes* & $26.6(10.3$ to 42.9$)$ & 14.1 (4.1 to 24.1$)$ \\
\hline \multicolumn{3}{|l|}{ CD8+ lymphocyte count (cells $/ \mathrm{mm}^{3}$ ) } \\
\hline Mean level in 4/96 & 863.2 (813.0 to 913.4 ) & 940.5 (913.0 to 967.9$)$ \\
\hline Slope per year $10 / 94-4 / 96$ & $6.5(-29.2$ to 42.3$)$ & $37.3(17.1$ to 57.4$)$ \\
\hline Slope per year $4 / 96-9 / 99$ & $-12.5(-24.6$ to -0.6$)$ & $-22.1(-28.8$ to -15.5$)$ \\
\hline Difference of slopes* & $-19.1(-62.3$ to 24.1$)$ & $-59.4(-83.8$ to -35.0$)$ \\
\hline
\end{tabular}

two or more nucleoside RTIs (54\%). Among all women receiving HAART, the most commonly reported protease inhibitors between 4/99 and 9/99 were nelfinavir (38\%) and indinavir (24\%); the most common nucleoside RTI combination was zidovudine/lamivudine $(31 \%)$. The reported use of these agents began to increase shortly after their approval by the FDA.

Other medications used to treat or prevent opportunistic infections also significantly increased over the period of study. The reported use of Pneumocystis carinii pneumonia (PCP) therapies (Bactrim (TMP-SMX), dapsone, pentamidine, atovaquone, daraprim, clindamycin, primaquine, trimextrate, and fansidar) from before to after 4/96 increased from 54\% to $61 \%$. Over the same period, antimycobacterial treatment (azithromycin, cripo, clarithromycin, clofazimine, amikacin, rifabutin, ethambutol, isoniazid, rifampin, and pyrazinamide) increased from $12 \%$ to $22 \%$, acyclovir use increased from $15 \%$ to $30 \%$, and antifungal therapy (ketoconazole, itraconazole, and fluconazole, ampho B, nystatin, mycelex, monistat, and terazol) increased from $31 \%$ to $44 \%$.

Table 2 details the trends in reported clinical AIDS (among women not reporting AIDS at baseline) and mortality in six month intervals. During the course of follow up, the incidence of clinical AIDS showed a slight (although non-significant) rise over the first year 4/95 to 3/96 ( 12.09 to 14.50 cases per 100 person years), but then showed a significant $14 \%$ semiannual decline over time to 5.07 cases per 100 person years between $4 / 98$ and 3/99. When excluding the data prior to 4/96, the estimated decline was $23 \%$ per semester. The most common AIDS defining condition reported in both the first and last intervals was oesophageal candidiasis.
The overall mortality rate also declined over the course of follow up (table 2). The mortality rate among the women reporting clinical AIDS at baseline was much higher than for those not reporting clinical AIDS at baseline. The mortality rate showed a strong, significant decline by $17 \%$ per semester among those reporting clinical AIDS at baseline from 4/95 to 3/99. In contrast, the mortality rate over the same time period among those women not reporting clinical AIDS at baseline did not show a statistically significant decline $(-5 \%$ per semester). When excluding the data in the first year (4/95-3/ 96), however, there was a statistically significant $11 \%$ semiannual decline in mortality between 4/96 and 3/99.

Table 3 describes the longitudinal trends estimated using random effects models for $\mathrm{CD} 4+$ and $\mathrm{CD} 8+$ lymphocyte counts among those with at least one visit after 4/96. The women reporting clinical AIDS at baseline had lower mean levels of both markers throughout the entire follow up period. The annual CD4+ decline of 19.4 cells $/ \mathrm{mm}^{3}$ per year before 4/96 improved to a 7.2 cells/ $\mathrm{mm}^{3}$ annual increase between $4 / 96$ and 9/99. The CD8+ slope in the period before 4/96 was not distinguishable from zero, nor from the CD8+ slope between $4 / 96$ and 9/99, but did decline in the latter period by 12.5 cells/year (95\% CI -24.5 to -0.6 ). Qualitatively similar trends were found among the women not reporting clinical AIDS at baseline. The annual CD4+ decline of 13.0 cells $/ \mathrm{mm}^{3}$ per year before $4 / 96$ also changed to a 1.1 cells/ $\mathrm{mm}^{3}$ annual increase after 4/96. CD8+ increases before 4/96, and declines after 4/96 were larger in magnitude and significance.

The contrast of the greater CD4+ increases among those with AIDS as compared with those not reporting AIDS at baseline was investigated further by stratifying the AIDS free

Table 4 CD4+ Lymphocyte characteristics and HAART use of AIDS free women stratified by baseline CD4+ lymphocyte count

\begin{tabular}{|c|c|c|c|c|c|c|}
\hline \multirow[b]{2}{*}{ Time period } & \multicolumn{2}{|c|}{ Baseline CD4 <200 $(n=224)$} & \multicolumn{2}{|c|}{$\begin{array}{l}200 \leqslant \text { Baseline CD4 }<500 \\
(n=573)\end{array}$} & \multicolumn{2}{|c|}{ Baseline CD4 $\geqslant 500(n=364)$} \\
\hline & HAART use & $\begin{array}{l}\text { Mean CD4 } \\
\text { (cells } / \mathrm{mm}^{3} \text { ) }\end{array}$ & HAART use & $\begin{array}{l}\text { Mean CD4 } \\
\text { (cells } / \mathrm{mm}^{3} \text { ) }\end{array}$ & HAART use & Mean CD4 (cells $/ \mathrm{mm}^{3}$ ) \\
\hline $10 / 94-3 / 95$ & $1.4 \%$ & 112 & $0.0 \%$ & 344 & $0.0 \%$ & 733 \\
\hline $4 / 95-9 / 95$ & $0.5 \%$ & 116 & $0.6 \%$ & 361 & $0.0 \%$ & 703 \\
\hline $10 / 95-3 / 96$ & $2.0 \%$ & 96 & $0.9 \%$ & 352 & $0.0 \%$ & 696 \\
\hline $4 / 96-9 / 96$ & $16.8 \%$ & 125 & $5.9 \%$ & 358 & $3.0 \%$ & 664 \\
\hline $10 / 96-3 / 97$ & $52.9 \%$ & 151 & $23.4 \%$ & 372 & $11.1 \%$ & 684 \\
\hline 4/97-9/97 & $61.7 \%$ & 173 & $39.1 \%$ & 369 & $19.0 \%$ & 595 \\
\hline $10 / 97-3 / 98$ & $62.3 \%$ & 188 & $46.8 \%$ & 358 & $23.8 \%$ & 618 \\
\hline 4/98-9/98 & $63.1 \%$ & 211 & $46.7 \%$ & 370 & $28.7 \%$ & 607 \\
\hline $10 / 98-3 / 99$ & $66.0 \%$ & 231 & $56.0 \%$ & 383 & $35.7 \%$ & 592 \\
\hline 4/99-9/99 & $73.9 \%$ & 223 & $59.7 \%$ & 388 & $35.0 \%$ & 586 \\
\hline CD4+ slope per year $10 / 94-4 / 96$ * & & $4.6(-9.2$ to 18.4$)$ & & $2.7(-8.5$ to 13.8$)$ & & $-58.8(-80.4$ to -37.3$)$ \\
\hline CD4+ slope per year 4/96-9/99* & & $35.6(30.6$ to 40.5$)$ & & $8.6(5.0$ to 12.3$)$ & & $-27.8(-34.6$ to -21.0$)$ \\
\hline Difference of slopes ${ }^{*} \dagger$ & & $31.0(13.9$ to 48.1$)$ & & $5.9(-7.5$ to 19.3$)$ & & $31.0(5.2$ to 56.8$)$ \\
\hline
\end{tabular}




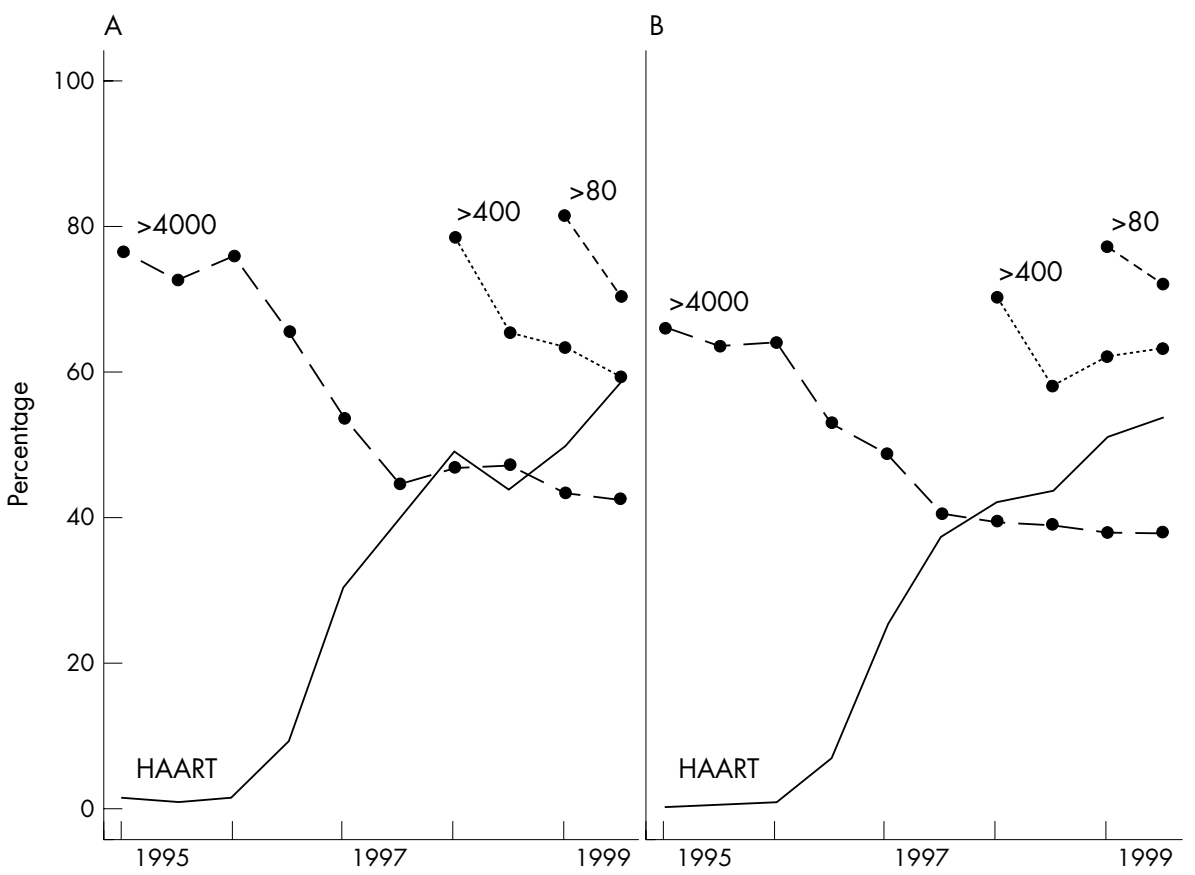

Figure 1 Temporal trends in plasma HIV RNA among women with (A) and without (B) self reported AIDS at baseline. The solid lines without points depict the percentage of women reporting HAART. The lines with points describe the percentage of women with HIV RNA above 4000 $\mathrm{cps} / \mathrm{ml}$ (solid lines) throughout the entire follow up period, and the percentage above $400 \mathrm{cps} / \mathrm{ml}$ (dotted lines) and $80 \mathrm{cps} / \mathrm{ml}$ (dashed lines) at later times when more sensitive assays were used. Data are plotted at midpoint of calendar.

women by their baseline CD4+ lymphocyte count (table 4). The women with lower CD4+ lymphocyte counts were more likely to receive HAART when it became available, consistent with our prior reports documenting selection by indication effects. $^{7}$ The response to treatment was different for each CD4+ strata, with those women in the lowest CD4+ strata showing the best overall trends in CD4+ response after 4/96 (+35.6 cells/year). Furthermore, the women with the highest CD4+ lymphocyte counts, and the lowest reported use of therapy, showed a decline after 4/96 ( -27.8 cells/year) that was significantly slower than the decline prior to $4 / 96$ ( -58.8 cells/ year).

Figure 1 depicts changes in plasma HIV RNA and changes in HAART, stratified among those women with AIDS at baseline (fig 1A) and those without AIDS at baseline (fig 1B). The figure describes the percentage of women with HIV RNA above $4000 \mathrm{cps} / \mathrm{ml}$ throughout the entire follow up period, and the percentage above $400 \mathrm{cps} / \mathrm{ml}$ and $80 \mathrm{cps} / \mathrm{ml}$ at later times when more sensitive assays were used. Prior to $4 / 96$, the percentage of samples that were above $4000 \mathrm{cps} / \mathrm{ml}$ was unchanged, and the women reporting clinical AIDS at baseline had a higher percentage with HIV RNA (75\%) above $4000 \mathrm{cps} / \mathrm{ml}$ than those women not reporting AIDS (64\%). Beginning with the data collected after $4 / 96$, the percentage of women above $4000 \mathrm{cps} / \mathrm{ml}$ dramatically declined in both groups up to mid-1997. After that time, this percentage was stable at approximately $40 \%$ for both groups.

\section{DISCUSSION}

This study showed a decline in mortality and self reported AIDS, as well as favourable trends in markers of HIV disease progression, over the time that HAART became available to women in the WIHS. These descriptions are important for evaluating how newly available therapies have had an impact on the clinical and laboratory manifestations of HIV-1 infection in a population-based setting outside of clinical trials. Prospective cohort studies that comprehensively characterise a broad range of people over time provide the ideal setting to describe these trends. Our cohort is closed to enrollment of new individuals; thus, without treatments, the natural history of HIV infection would provide an expectation that the average CD4+ cell counts decline and AIDS incidence and mortality increase. Taken in this context, the attenuation of marker decline over time and reducing incidence of clinical outcomes are even more significant.

From a public health perspective, it is particularly important to record changes in HIV related clinical outcomes in women who reflect recent demographic characteristics of HIV infection in the United States, have more severe disease, and are receiving HAART. Consistent with a number of prior reports, the results of our study show trends in women that are both qualitatively and quantitatively similar to the trends described in HIV infected men. For example, in the HIV outpatient study that consisted of participants with CD4+ lymphocyte counts below 100 cells $/ \mathrm{mm}^{3}$, the mortality rate declined from 29.4 in 1995 to 8.8 cases per 100 person year in 1997. ${ }^{18}$

Our study is one of the first, however, to describe the dramatic population level changes in HIV RNA levels occurring with the introduction of HAART. The high proportion of WIHS women with HIV RNA levels below the NASBA limit of detection used in this study (4000 copies/ml) indicates a potential benefit of retesting using more sensitive HIV RNA assays. The multi-year $-70^{\circ} \mathrm{C}$ storage of plasma in the WIHS for ongoing retesting with more sensitive assays demonstrates the utility of maintaining specimens in anticipation of future advances in technology.

As shown in table 4, the strongest CD4+ lymphocyte responses occurred among those who began with the lowest CD4+ lymphocyte counts. Because of the "selection by indication" effects determining who receives treatment, ${ }^{7}$ this group also had the highest proportion using HAART. The improvements in CD4+ lymphocyte counts in this group are consistent with changes reported by others in controlled studies of people with very low CD4+ lymphocyte counts ${ }^{49}$; little clinical data exist for comparing the changes among those with less impaired immune systems. However, our data show the public health impact of treatments when administered in the population, complementing the findings from 
clinical trials. HAART use of up to $60 \%$ among those with CD4+ lymphocyte counts between 200 and 500 at baseline led to negligible changes in CD4+ trajectories; among those with counts above 500, CD4+ lymphocyte counts continued to decline, although at a slower rate. CD4 slopes before April 1996 may underestimate the rate of change in the general population, as we have excluded women with the most rapid rates of $\mathrm{CD} 4$ decline who died before the introduction of HAART.

The HIV RNA trends in 1997 to 1999, and the stabilisation of AIDS incidence and mortality rates after April 1998, show that the largest impact of HAART in this cohort has perhaps already occurred. There exists a substantial pool of women who have not yet started HAART in the cohort because of a number of factors, ${ }^{5}$ including that their disease has not yet progressed to the level that treatment is recommended, possible problems with access, and the women's decision to begin treatment. Individually, some of these women will benefit from initiating potent treatment early while others are likely to receive the optimal benefit from delaying the start of therapy. From a population perspective, women who are most ill and at highest risk for clinical disease and death have generally started treatment, expanding the use of HAART is likely to have a less dramatic impact on reducing HIV RNA levels and on the incidence of clinical disease and death.

We have attributed much of the observed longitudinal improvement in lymphocyte subsets and viral RNA levels to the introduction and expanded use of HAART. However, alternative explanations may be possible. Firstly, the introduction of other treatments and changing patterns of use of existing therapies could account for some of the positive longitudinal trends. For example, stavudine was approved by the FDA in mid-1994, and lamivudine was approved in late 1995. Previously published reports have attributed improvements in survival time to the introduction of these drugs, ${ }^{20}$ which occurred just before and, in part, concurrently with the introduction of protease inhibitors; these changes are therefore difficult to distinguish. We have also described increasing use of prophylaxis for opportunistic infections (that is, PCP, Mycobacterium avium complex, herpes simplex viruses and varicella zoster virus) in the same era as the expanded use of HAART, which might also account for some of the decline in the incidence of AIDS.

Other characteristics of this work relate to our use of self reported AIDS; confirmation of diagnoses via medical record abstraction and matching to AIDS registries is ongoing. Reviews of AIDS self reports with confirmations from registry data, ${ }^{50}$ and the strong association of incident self reported AIDS with HIV RNA and CD4+ lymphocyte counts ${ }^{51}$ provide encouraging evidence of the overall reliability of these self reported data. However, it is possible that some of the decline in self reported AIDS over time may be because of changes in reporting behaviour-either a decrease in overreporting or an increase in underreporting.

Participant survival bias could be another potential explanation for the observed declines in death and self reported AIDS. The initially high prevalence of AIDS and subsequent death would remove the least healthy individuals who have been infected the longest. The resulting cohort at later times would be substantially healthier, thereby leading to lower numbers of subsequent events. ${ }^{52}$ One solution to this problem is to analyse data from a cohort of persons with a known date of HIV infection, and then take the time that has passed from infection to the date of study in consideration. ${ }^{22}$ Unfortunately, observation of incident HIV infection is technically difficult, particularly in a population such as US women, among whom HIV infection is a relatively infrequent event. Recently, methods have been introduced to overcome the uncertainty in time of seroconversion through the use of information contained in disease progression markers ${ }^{53}$; investigations with WIHS data are continuing.
Lastly, we have not considered other important issues related to the use of antiretroviral therapy, such as access to care, adherence to treatment, and development of drug resistance. Most of the WIHS participants reported having medical insurance (including either government assisted public insurance, private insurance, or combinations) and having a primary care provider, ${ }^{54}$ although the WIHS does not provide medical care beyond referrals for abnormal findings. This suggests that access to antiretroviral therapy among these women was not a major impediment to use. In the general population, however, there are often substantial barriers to care ${ }^{54-56}$ that may account for some of the heterogeneity of survival times from AIDS. ${ }^{23}$ Data on treatment adherence ( $\mathrm{T}$ Wilson, et al, International AIDS Conference, Durban, South Africa 2000) and drug related resistance (RM Grant, et al, 5th Workshop on Drug Resistance and Treatment Strategies, 2001) in the WIHS are currently being investigated.

In summary, our data provide important and encouraging evidence that the public health gains seen in other populations of HIV infected people in the US are also experienced by HIV infected US women. It will be important to continue monitoring cohorts such as the WIHS for further trends in HIV disease outcomes as new therapies are approved and come into widespread use. Furthermore, cohort studies offer the opportunity to detect and track the occurrence of long term side effects (such as ART induced lipodystrophy) or treatment failure, perhaps as a result of the development of viral resistance. Follow up over longer time periods will be particularly important, as current clinical trials are of short ( $1-2$ year) duration with little long term monitoring.

\section{ACKNOWLEDGEMENTS}

Data in this manuscript were collected by the Women's Interagency HIV Study (WIHS) Collaborative Study Group with centres (Principal Investigators) at New York City/Bronx Consortium (Kathryn Anastos); Brooklyn, NY (Howard Minkoff); Washington DC Metropolitan Consortium (Mary Young); The Connie Wofsy Study Consortium (Ruth Greenblatt, Herminia Palacio); Los Angeles County/Southern California Consortium (Alexandra Levine); Chicago Consortium (Mardge Cohen); Data Coordinating Center (Alvaro Muñoz, Stephen $\mathrm{J}$ Gange). Participating institutions approved this study and consent forms provided to study participants.

We gratefully acknowledge Lynn Kirstein, Lorie Benning, Mary Elizabeth Gore, and Galen Mayfield for their leadership and innovation in the management of WIHS data, and Alexandra Levine for her constructive review and suggestions.

\section{Authors' affiliations}

S J Gange, Y Barrón, A Muñoz, Department of Epidemiology, Johns Hopkins School of Hygiene and Public Health, Baltimore, USA

R M Greenblatt, Departments of Medicine and Epidemiology, University of California at San Francisco, USA

K Anastos, Montefiore Medical Center, New York, USA

H Minkoff, Department of Obstetrics and Gynecology, Maimonides

Medical Center and SUNY Health Sciences Center at Brooklyn, New York, USA

M Young, Georgetown University Medical Center, Washington, USA

A Kovacs, Division of Pediatric Infectious Diseases and Comprehensive

Maternal-Child HIV Management and Research Center, Los Angeles

County, and the University of Southern California School of Medicine, Los Angeles, USA

M Cohen, Cook County Hospital, Chicago, USA

W A Meyer III, Quest Diagnostics Incorporated, Baltimore, USA

\section{REFERENCES}

1 Hammer S, Squires K, Hughes M, et al. A controlled trial of two nucleoside analogues plus indinavir in persons with human immunodeficiency virus infection and CD4 lymphocyte counts of 200 pe cubic millimeter or less. AIDS Clinical Trials Group Study Section. N Engl J Med 1997;337:725-33

2 Gulick R, Mellors J, Havlir D, et al. Treatment with indinavir, zidovudine and lamivudine in adults with human immunodeficiency virus infection and prior antiretroviral therapy. N Engl J Med 1997;337:734-9. 
3 Schapiro J, Winters M. Stewart $F$, et al. The effect of high-dose saquinavir on viral load and CD4+ T-cell counts in HIV-infected patients. Ann Intern Med 1996;1 24:1039-50.

4 Muñoz A, Gange S and Jacobson L. Distinguishing efficacy, individual effectiveness and population effectiveness of therapies. AIDS 2000;14:754-6.

5 Cook JA, Cohen MH, Grey D, et al. Penetration and predictors of use of highly active antiretroviral therapy in a cohort of HIV-seropositive women. Am J Public Health (in press).

6 Bing EG, Kilbourne AM, Brooks RA, et al. Protease inhibitor use among a community sample of people with HIV disease. J Acquired Immune Deficiency Synd Hum Retrovir 1999;20:474-80.

7 Ahdieh L, Gange SJ, Greenblatt R, et al. Selection by indication of potent antiretroviral therapy usage in a large cohort of HIV-infected women. Am J Epidemiol 2000;152:923-33.

8 Graham NMH, Zeger SL, Park LP, et al. Effect of zidovudine and Pneumocystis carinii pneumonia prophylaxis on progression of HIV-1 infection to AIDS. Lancet 1991;338:265-9

9 Robins JM, Hernan MA, Brumback B. Marginal structural models and causal inference in epidemiology. Epidemiology 2000;1 1:550-60.

10 Vella S, Giuliano M, Pezzotti P, et al. Survival of zidovudine-treated patients with AIDS compared with that of contemporary untreated patients. Italian Zidovudine Evaluation Group. JAMA 1992;267:12326.

11 Phillips AN, Grabar S, Tassie J-M, et al. Use of observational database to evaluate the effectiveness of antiretroviral therapy for HIV infection: comparison of cohort studies with randomized trials. AIDS $1999 ; 13: 2075-82$

12 Sabin CA. Assessing the impact of highly active antiretroviral therapy on AIDS and death. AIDS 1999:13:2165-6.

13 Muñoz A, Hoover DR. Use of cohort studies for evaluating AIDS therapies. In: Finkelstein DM, Schoenfeld DA, eds. AIDS clinical trials. New York: Wiley-Liss, 1995:423-46.

14 Gail MH. Use of observational data for evaluating AIDS therapies. In: Finkelstein DM, Schoenfeld DA, eds. AIDS Clinical Trials. New York: Wiley-Liss, 1995:403-22.

15 Hoover DR, Muñoz A, He Y, et al. The effectiveness of interventions on incubation of AIDS as measured by secular increases within a population. Stat Med 1994;13:2127-39.

16 Egger $M$, Hirschel B, Francioli $P$, et al. Impact of new antiretroviral combination therapies in HIV infected patients in Switzerland: prospective multicentre study. BM 1997;315:1194-9.

17 Mocroft A, Vella S, Benfield TL, et al. Changing patterns of mortality across Europe in patients infected with HIV-1: EuroSIDA Study Group. Lancet 1998:352:1725-30.

18 Paella FJ, Delaney KM, Moorman AC, et al. Declining morbidity and mortality among patients with advanced human immunodeficiency virus infection. N Engl J Med 1998;338:853-60.

19 Veugelers PJ, Cornelisse PGA, Craib KJP, et al. Models of survival in HIV infection and their use in the quantification of treatment benefits. Am J Epidemiol 1998;148:487-97.

20 Hogg RS, O'Shaughnessy MV, Gataric N, et al. Decline in deaths from AIDS due to new antiretrovirals. Lancet 1997; 349:1294.

21 Ledergerber B, Egger M. Opravil M, et al. Clinical progression and virological failure on highly active antiretroviral therapy in HIV-1 patients: a prospective cohort study: Swiss HIV Cohort Study. Lancet 1999;353:863-8.

22 Detels R, Muñoz A, McFarlane G, et al. Effectiveness of potent antiretroviral therapy on time to AIDS and death in men with known HIV infection duration. JAMA 1998:280:1497-503

23 Lee LM, Karon JM, Selik R, et al. Survival after AIDS diagnosis in adolescents and adults during the treatment era, United States, 1984-1997. JAMA 2001;285:1308-15.

24 Pezzotti P, Napoli PA, Acciai S, et al. Increasing survival time after AIDS in Italy: the role of new combination antiretroviral therapies. AIDS 1999; 13:249-55.

25 Rosenberg PS. Scope of the AIDS epidemic in the United States. Science 1995;270:1372-5

26 Rosenberg PS, Biggar RJ. Trends in HIV incidence among young adults in the United States. JAMA 1998;279:1894-9.

27 United States Centers for Disease Control and Prevention HIV/AIDS Surveillance Report: midyear edition 1998;10:5-21.

28 United States Centers for Disease Control and Prevention. HIV/AIDS Surveillance Supplemental Report. Characteristics of persons living with AIDS at the end of 1997. MMWR 1999;5:4-6.

29 Porta D, Rapiti E, Forastiere F, et al. Changes in survival among people with AIDS in Lazio, Italy from 1993 to 1998. AIDS 1999;13:2125-31.
30 Poundstone KE, Chaisson RE, Moore RD. Differences in HIV disease progression by injection drug use and by sex in the era of highly active antiretroviral therapy. AIDS 2001;15:1115-23.

31 Farzadegan $\mathbf{H}$, Hoover D, Astemborski J, et al. Sex differences in HIV-1 viral load and progression to AIDS. Lancet 1998:352:1510-14.

32 Anastos K, Gange SJ, Lau B, et al. Association of race and gender with HIV-1 RNA levels and immunologic progression. J Acquir Immune Defic Syndr 2000;24:218-26.

33 Sterling TR, Vlahov D, Astemborski J, et al. Initial plasma HIV-1 RNA levels and progression to AIDS in women and men. N Engl J Med 2001:344:720-5

34 Ho D, Neumann AU, Perelson AS, et al. Rapid turnover of plasma virions and CD4 lymphocytes in HIV-1 infection. Nature 1995;373:123-6.

35 Wei X, Ghosh SK, Taylor ME, et al. Viral dynamics in human immunodeficiency virus type 1 infection. Nature 1995;373:117-22.

36 Greenblatt R, Ameli N, Grant RM, et al. Impact of the ovulatory cycle on virologic and immunologic markers in HIV-infected women. J Infect Dis 2000;181:82-90.

37 Bozzette SA, Berry SH, Duan N, et al. The care of HIV-infected adults in the United States. HIV Cost and Services Utilization Study Consortium. N Engl J Med 1998; 339:1897-904.

38 Kleeberger CA, Phair JP, Strathdee SA, et al. Determinants of heterogeneous adherence to HIV-antiretroviral therapies in the Multicenter AIDS Cohort Study. J Acquir Immune Defic Syndr 2001;26:82-92.

39 Shapiro MF, Morton SC, McCaffrey DF, et al. Variations in the care of HIV-infected adults in the United States: results From the HIV Cost Services Utilization Study. JAMA 1999;281:2305-15.

40 Rapiti E, Porta D, Forastiere F, et al. Socioeconomic status and survival of persons with AIDS before and after the introduction of highly active antiretroviral therapy. Lazio AIDS Surveillance Collaborative Group. Epidemiology 2000;11:496-501.

41 Marx R, Hirozawa A, Soskolne V, et al. Barriers to getting needed services for Ryan White CARE clients. AIDS Care 2001;13:233-42.

42 Barkan SE, Melnick SL, Preston-Martin S, et al. The Women's Interagency HIV Study (WIHS): Design, methods, sample, cohor characteristics and comparison with reported AIDS cases in US women. Epidemiology 1998;9:117-25.

43 Centers for Disease Control and Prevention. 1993 Revised classification system for HIV infection and expanded surveillance case definition for AIDS among adolescents and adults. MMWR 1992;41 (no RR-17).

44 Hessol NA, Schneider M, Greenblatt RM, et al. Retention of women enrolled in a prospective study of HIV infection: Impact of race, unstable housing, and use of HIV therapy. Am J Epidemiol 2001;54:563-73.

45 Feinberg MB, Carpenter C, Fauci A, et al. Guidelines for the use of antiretroviral agents in HIV-infected adults and adolescents. Ann Intern Med. 1998;128:1079-100.

46 Breslow NE, Day NE. Statistical methods in cancer research. Volume II The design and analysis of cohort studies. Lyon: IARC Scientific Publications, 1987

47 Laird NM, Ware JH. Random-effects models for longitudinal data. Biometrics 1982;38:963-74

48 Diggle PJ, Liang KY, Zeger SL. Analysis of longitudinal data. Oxford: Oxford University Press, 1994

49 Autran B, Carcelain G, Li TS, et al. Positive effects of combined antiretroviral therapy on CD4+ T-cell homeostasis and function in advanced HIV disease. Science 1997;277:112-16.

50 Hessol N, Schwarcz S, Ameli N, et al. Accuracy of self-reports of AIDS and AIDS-related conditions in women. Am J Epidemiol 2001;153:1128-33.

51 Hessol NA, Ameli N, Anastos K, et al. Factors associated with incident self-reported AIDS among women in the Women's Interagency HIV Study (WIHS). AIDS Res Hum Retroviruses 2000;16:1105-12.

52 Glesby MJ, Hoover DR. Survivor treatment selection bias in observational studies: Examples from the AIDS literature. Ann Intern Med 1996; 124:999-1005

53 Tarwater PM, Mellors J, Gore ME, et al. Methods to assess population effectiveness of therapies in HIV-1 incident and prevalent cohorts. Am Epidemiol 2001;54:675-81.

54 Palacio $\mathbf{H}$, Shiboski CH, Yelin EH, et al. Access to and utilization of primary care services among HIV-infected women. J Acquir Immune Defi Syndr Hum Retrovirol 1999;21:293-300.

55 Heckman TG, Somlai AM, Peters J, et al. Barriers to care among persons living with HIV/AIDS in urban and rural areas. AIDS Care $1998 \cdot 10 \cdot 365-75$

56 Anderson R, Bozzette S, Shapiro M, et al. Access of vulnerable groups to antiretroviral therapy among persons in care for HIV disease in the United States. Health Serv Res 2000;35:389-416. 